\title{
Análise comparativa da flexibilidade de instrumentos endodônticos, submetidos ou não a tratamento térmico
}

\section{Comparative analysis of the flexibility of endodontic instruments, submitted or not to thermal treatment}

\author{
Sérgio Koiti KAMEI* \\ Marcelo dos SANTOS** \\ Antônio Carlos BOMBANA***
}

\begin{abstract}
KAMEI, S. K.; SANTOS, M.; BOMBANA, A. C. Análise comparativa da flexibilidade de instrumentos endodônticos, submetidos ou não a tratamento térmico. Pesqui Odontol Bras, v. 14, n. 3, p. 213-218, jul./set. 2000.

O presente estudo buscou avaliar a flexibilidade de dois tipos de limas: tipo K e Flexofile ${ }^{\circledR}$ de diferentes números, submetidas ou não a um tratamento térmico recristalizador. Para tanto, utilizaram-se 144 limas que foram submetidas ao ensaio de flexão em um troptômetro e uma célula de carga. Os resultados evidenciaram que o tratamento térmico recristalizador foi capaz de provocar a redução na resistência à flexão das limas tipo K de 56,78 a 82,06\%. Nas limas Flexofile $^{\circledR}$, a diminuição da resistência à flexão foi da ordem de 1,94 a $50,60 \%$, quando comparadas com as Flexofile ${ }^{\circledR}$ nãotratadas. Concluiu-se que o tratamento térmico recristalizador, como o proposto, reduz a resistência à flexão das limas tipo K de forma significativa, e das limas tipo Flexofile ${ }^{\circledR}$ de maneira pouco sensivel.
\end{abstract}

UNITERMOS: Instrumentos odontológicos; Preparo de canal radicular.

\section{INTRODUÇÃO}

O sucesso endodôntico deriva da obturação definitiva e hermética do canal cirúrgico, possibilitado pelo adequado preparo do canal radicular onde se procura obter o máximo de limpeza e uma melhor modelagem. Entretanto, em certas ocasiões, o canal radicular apresenta-se visivelmente encurvado, detalhe anatômico que dificulta a adaptação dos instrumentos às paredes dentinárias e suas ações associadas à manutenção da forma original do canal radicular.

A transposição desse obstáculo está subordinada ao conhecimento, ao domínio técnico, ao adestramento profissional, à experiência clínica e às propriedades físico-mecânicas dos instrumentos.

A preocupação em solucionar esse problema pode ser notada pela diversidade de técnicas e pelos estudos de diversos autore s $^{1,14,15,20}$.

A obtenção de uma peça metálica como a lima é produto final de um processo de conformação plástica em que se aplicam tensões por trabalhos a frio (abaixo da temperatura de recristalização do metal). Como conseqüência desse trabalho a frio, ocorre o fenômeno de encruamento do metal que acarreta modificações em algumas propriedades físicas (diminuição da densidade e condutibilidade elétrica) e mecânicas (maior limite de escoamento, resistência e maior dureza), acorde BRESCIANI et al. ${ }^{5}$ (1991).

$\mathrm{Na}$ busca de alternativas para as dificuldades clínicas criadas pela manufatura dos instrumentos, alguns caminhos foram propostos como o das alterações das ligas metálicas ${ }^{8,9,10,19}$, da geometria da seção transversal ${ }^{2,11,16,18}$, bem como os dos tratamentos térmicos ${ }^{12,17}$.

Por meio do tratamento térmico recristalizador, é possivel restabelecer as propriedades físico-mecânicas provocadas pelo encruamento. O tratamento térmico reorganiza a estrutura cristalina do metal aliviando o nivel de energia interna de acordo com os trabalhos de MANGONON; THOMAS ${ }^{13}$ (1970); CHIAVERINI ${ }^{7}$ (1986); ELIAS et al. ${ }^{12}$ (1993); SANTOS $^{17}$ (1994).

Assim, foi proposta deste trabalho avaliar a flexão de instrumentos tipo K e Flexofile ${ }^{\circledast}$ de números 15,25 e 30 submetidos a um tratamento térmico recristalizador comparada com a de outros, nãotratados.

\footnotetext{
* Professor Mestre da Disciplina de Endodontia do Curso de Odontologia da Universidade Metodista de São Paulo.

** Professor Doutor; *** Professor Associado - Disciplina de Endodontia da Faculdade de Odontologia da USP.
} 
KAMEI, S. K.; SANTOS, M.; BOMBANA, A. C. Análise comparativa da flexibilidade de instrumentos endodônticos, submetidos ou não a tratamento térmico. Pesqui Odontol Bras, v. 14, n. 3, p. 213-218, jul./set. 2000.

\section{MATERIAL E MÉTODO}

Foram utilizadas 144 limas endodônticas: 72 limas tipo K, marca Maillefer e 72 limas tipo Flexofi$1 \mathrm{e}^{\circledR}$, marca Maillefer.

Inicialmente, as 72 limas de cada tipo foram submetidas a exame visual em um perfilômetro. Posicionadas sob a luz do projetor, as linhas-guia do aparelho percorriam as limas indicando no mostrador digital a ausência de deformações plásticas permanentes; qualificadas nesse controle, as limas eram levadas para o ensaio de flexão.

Para realizar-se o ensaio de flexão, adaptou-se o troptômetro $^{17}$, aumentando-se a área de sua base de sustentação e adicionando-se uma guia metálica, de modo a permitir o posicionamento horizontal do mandril de Jacobs do troptômetro e da célula de carga em plano paralelo à base do equipamento.

A fixação das limas era obtida após a remoção de seus cabos com o auxílio de um alicate de corte. Em seguida, as limas recebiam uma marca com tinta permanente a $23 \mathrm{~mm}$ de $\mathrm{D}_{0}$, fixadas horizontalmente no nivel da marca ao mandril de Jacobs em posição paralela à escala de ângulos do troptômetro.

A célula de carga foi nivelada sobre a base de sustentação e, sob o mandril do troptômetro, a lima entrava em contato com o sensor da célula de carga de modo a indicar $0 \mathrm{~g}$ no mostrador digital. A escala do troptômetro era configurada para $0^{\circ}$.

Ao acionar-se a manivela das engrenagens, o mandril de Jacobs movimentava-se no sentido horário, até os ângulos de $30^{\circ}, 45^{\circ}$ e $60^{\circ}$ sucessivamente, indicados pela escala do troptômetro. A cada uma dessas angulações, anotava-se o maior valor da carga, em gramas, indicada no mostrador digital da célula de carga.

A seguir, as amostras de instrumentos restantes foram submetidas ao tratamento térmico recristalizador em um forno de recozimento à proporção de doze unidades de cada número e tipo ${ }^{17}$.

As limas foram confinadas em 6 tubos de quartzo com $8 \mathrm{~mm}$ de diâmetro interno, 1,5 $\mathrm{mm}$ de espessura de parede e $200 \mathrm{~mm}$ de comprimento e hermeticamente fechadas com o auxílio de um maçarico de acetileno-oxigênio. Os tubos selados foram introduzidos em um forno de recozimento à temperatura de $600^{\circ} \mathrm{C}$ por um período de 1 hora. Em seguida, foram removidos do forno e deixados à temperatura ambiente até o completo resfriamento. Posteriormente, os tubos foram rompidos e as limas submetidas a dois ensaios.
O primeiro consistia na observação visual de cada lima no perfilômetro, acerca da integridade e ausência de deformações plásticas permanentes. Os instrumentos aprovados nessa observação eram submetidos ao ensaio de flexão, de acordo com a metodologia já descrita.

Após a tabulação dos dados, estes foram submetidos à análise estatística pertinente com um grau de exigência de $5 \%(\alpha=0,05)$.

\section{RESULTADOS}

Os valores médios das cargas, em gramas, do ensaio de flexão para os ângulos de $30^{\circ}, 45^{\circ}$ e $60^{\circ}$ das limas tipo K e Flexofile ${ }^{\circledast}$ de números 15,25 e 30 tratadas e não-tratadas e o percentual de redução da carga após o tratamento térmico recristalizador encontram-se na Tabela 1.

A Tabela 2 apresenta as confrontações das diferenças entre as médias das cargas das limas nú-

TABELA 1 - Valores médios das cargas, em gramas, ao ensaio de flexão das limas tipo K e Flexofile ${ }^{\circledR}$ de números 15,25 e 30 tratadas e não-tratadas, nas angulações experimentais e o percentual de redução das cargas.

\begin{tabular}{|c|c|c|c|c|c|}
\hline $\begin{array}{l}\text { Lima - } \\
\text { número }\end{array}$ & $\begin{array}{c}\text { Lima }- \\
\text { tipo }\end{array}$ & Ângulo & $\begin{array}{c}\text { Não- } \\
\text { tratada-- } \\
\text { carga (g) }\end{array}$ & $\begin{array}{l}\text { Tratada - } \\
\text { carga }(\mathrm{g})\end{array}$ & $\begin{array}{c}\text { Redução } \\
\text { da carga } \\
(\%)\end{array}$ \\
\hline \multirow{6}{*}{15} & \multirow{3}{*}{ K } & $30^{\circ}$ & 110,91 & 46,66 & 57,92 \\
\hline & & $45^{\circ}$ & 247,00 & 58,00 & 76,51 \\
\hline & & $60^{\circ}$ & 367,91 & 66,00 & 82,06 \\
\hline & \multirow{3}{*}{$\begin{array}{l}\text { Flexo- } \\
\text { file }^{\circledR}\end{array}$} & $30^{\circ}$ & 72,75 & 55,58 & 23,60 \\
\hline & & $45^{\circ}$ & 166,66 & 92,25 & 44,64 \\
\hline & & $60^{\circ}$ & 248,16 & 122,58 & 50,60 \\
\hline \multirow{6}{*}{25} & \multirow{3}{*}{ K } & $30^{\circ}$ & 247,25 & 98,25 & 60,26 \\
\hline & & $45^{\circ}$ & 488,66 & 111,66 & 77,14 \\
\hline & & $60^{\circ}$ & 645,41 & 130,75 & 79,74 \\
\hline & \multirow{3}{*}{$\begin{array}{c}\text { Flexo- } \\
\text { file }^{\circledR}\end{array}$} & $30^{\circ}$ & 133,00 & 103,91 & 21,87 \\
\hline & & $45^{\circ}$ & 296,91 & 162,83 & 45,15 \\
\hline & & $60^{\circ}$ & 413,25 & 281,75 & 31,82 \\
\hline \multirow{6}{*}{30} & \multirow{3}{*}{$\mathrm{K}$} & $30^{\circ}$ & 304,83 & 131,66 & 56,80 \\
\hline & & $45^{\circ}$ & 600,41 & 253,25 & 57,82 \\
\hline & & $60^{\circ}$ & 838,08 & 362,16 & 56,78 \\
\hline & \multirow{3}{*}{$\begin{array}{c}\text { Flexo- } \\
\text { file }^{\circledR}\end{array}$} & $30^{\circ}$ & 197,16 & 193,33 & 1,94 \\
\hline & & $45^{\circ}$ & 438,41 & 307,91 & 29,76 \\
\hline & & $60^{\circ}$ & 606,16 & 378,33 & 37,58 \\
\hline
\end{tabular}


KAMEI, S. K.; SANTOS, M.; BOMBANA, A. C. Análise comparativa da flexibilidade de instrumentos endodônticos, submetidos ou não a tratamento térmico. Pesqui Odontol Bras, v. 14, n. 3, p. 213-218, jul./set. 2000.

TABELA 2 - Confrontação das diferenças entre as médias das cargas ao ensaio de flexão para os ângulos de $30^{\circ}, 45^{\circ}$ e $60^{\circ}$ das limas tipo K e Flexofile ${ }^{\circledR}$ de número 15 , submetidas ou não ao tratamento térmico e a comparação com o valor crítico calculado de Tukey a $5 \%$.

\begin{tabular}{|c|c|c|c|c|}
\hline Deflexão & & $\mathrm{KT}$ & $\mathrm{F}$ & FT \\
\hline \multirow{3}{*}{$30^{\circ}$} & $\mathrm{K}$ & $64,25 \mathrm{~s}$ & $38,17 \mathrm{~ns}$ & $55,33 \mathrm{~s}$ \\
\hline & KT & - & $26,08 \mathrm{~ns}$ & $8,92 \mathrm{~ns}$ \\
\hline & $\mathrm{F}$ & & - & $17,17 \mathrm{~ns}$ \\
\hline \multirow{4}{*}{$45^{\circ}$} & & KT & $\mathrm{F}$ & FT \\
\hline & $\mathrm{K}$ & $189,00 \mathrm{~s}$ & $80,33 \mathrm{~ns}$ & $154,75 \mathrm{~s}$ \\
\hline & $\mathrm{KT}$ & - & $108,67 \mathrm{~s}$ & $34,24 \mathrm{~ns}$ \\
\hline & $\mathrm{F}$ & & - & $74,42 \mathrm{~ns}$ \\
\hline \multirow{4}{*}{$60^{\circ}$} & & $\mathrm{KT}$ & $\mathrm{F}$ & FT \\
\hline & $\mathrm{K}$ & $301,92 \mathrm{~s}$ & $119,75 \mathrm{~ns}$ & $245,33 \mathrm{~s}$ \\
\hline & $\mathrm{KT}$ & - & $182,17 \mathrm{~s}$ & $56,58 \mathrm{~ns}$ \\
\hline & $\mathrm{F}$ & & - & $125,58 \mathrm{~ns}$ \\
\hline
\end{tabular}

Valor crítico de Tukey a $5 \%$ para $30^{\circ}=39,02$, para $45^{\circ}=85,51$ e para $60^{\circ}=125,62(\mathrm{~K}=$ tipo $\mathrm{K}$ não-tratada; $\mathrm{KT}=$ tipo $\mathrm{K}$ tratada;

$\mathrm{F}=$ Flexofile ${ }^{\circledR}$ não-tratada; FT $=$ Flexofile $^{\circledR}$ tratada; $\mathrm{ns}=$ não significante; $\mathrm{s}=$ significante).

mero 15 levadas à flexão de $30^{\circ}, 45^{\circ}$ e $60^{\circ}$, comparadas duas a duas.

A Tabela 3 apresenta as confrontações das diferenças entre as médias das cargas das limas número 25 levadas à flexão de $30^{\circ}, 45^{\circ}$ e $60^{\circ}$, comparadas duas a duas.

A Tabela 4 apresenta as confrontações das diferenças entre as médias das cargas das limas número 30 levadas à flexão de $30^{\circ}, 45^{\circ}$ e $60^{\circ}$, comparadas duas a duas.

\section{DISCUSSÃO}

Ao submeter-se limas tipo K e Flexofile ${ }^{\circledR}$ ao tratamento térmico recristalizador como proposto, nota-se que as limas tipo $\mathrm{K}$ foram bastante susceptiveis ao tratamento térmico, pois quando tratadas necessitaram de menor carga para serem fletidas para todos os números e ângulos e as diferenças entre os valores encontrados para as limas tipo $\mathrm{K}$ tratadas e as não-tratadas, para todas as situações, foram estatisticamente significativas ao nivel de $\alpha=5 \%$.

As limas Flexofile ${ }^{\circledR}$ não apresentaram alterações significativas sob o ponto de vista estatístico, quando se compararam os valores necessários para a flexão dos instrumentos tratados com os
TABELA 3 - Confrontação das diferenças entre as médias das cargas ao ensaio de flexão para os ângulos de $30^{\circ}, 45^{\circ}$ e $60^{\circ}$ das limas tipo K e Flexofile ${ }^{\circledR}$ de número 25 , submetidas ou não ao tratamento térmico e a comparação com o valor crítico calculado de Tukey a 5\%.

\begin{tabular}{c|c|c|c|c}
\hline \hline Deflexão & & $\mathrm{KT}$ & $\mathrm{F}$ & $\mathrm{FT}$ \\
\hline \multirow{3}{*}{$30^{\circ}$} & $\mathrm{K}$ & $149,00 \mathrm{~s}$ & $114,25 \mathrm{~s}$ & $143,33 \mathrm{~s}$ \\
\cline { 2 - 5 } & $\mathrm{KT}$ & - & $34,75 \mathrm{~ns}$ & $5,67 \mathrm{~ns}$ \\
\cline { 2 - 5 } & $\mathrm{F}$ & & - & $29,08 \mathrm{~ns}$ \\
\hline \multirow{4}{*}{$45^{\circ}$} & $\mathrm{K}$ & $377,00 \mathrm{~s}$ & $191,75 \mathrm{~s}$ & $325,83 \mathrm{~s}$ \\
\cline { 2 - 5 } & $\mathrm{KT}$ & - & $185,25 \mathrm{~s}$ & $51,17 \mathrm{~ns}$ \\
\cline { 2 - 5 } & $\mathrm{F}$ & & - & $134,08 \mathrm{~s}$ \\
\hline \multirow{4}{*}{$60^{\circ}$} & $\mathrm{K}$ & $514,66 \mathrm{~s}$ & $232,16 \mathrm{~ns}$ & $363,66 \mathrm{~s}$ \\
\cline { 2 - 5 } & $\mathrm{KT}$ & - & $282,50 \mathrm{~s}$ & $151,00 \mathrm{~ns}$ \\
\cline { 2 - 5 } & $\mathrm{F}$ & & - & $131,50 \mathrm{~ns}$ \\
\cline { 2 - 5 } & $\mathrm{KT}$ & $\mathrm{F}$ & $\mathrm{FT}$ \\
\hline \hline
\end{tabular}

Valor crítico de Tukey a $5 \%$ para $30^{\circ}=67,27$, para $45^{\circ}=89,05$ e para $60^{\circ}=260,35(\mathrm{~K}=$ tipo $\mathrm{K}$ não-tratada; $\mathrm{KT}=$ tipo $\mathrm{K}$ tratada;

$\mathrm{F}=$ Flexofile ${ }^{\circledR}$ não-tratada; FT $=$ Flexofile ${ }^{\circledR}$ tratada; $\mathrm{ns}=$ não significante; $\mathrm{s}=$ significante).

TABELA 4 - Confrontação das diferenças entre as médias das cargas ao ensaio de flexão para os ângulos de $30^{\circ}, 45^{\circ}$ e $60^{\circ}$ das limas tipo $\mathrm{K}$ e Flexofile ${ }^{\circledR}$ de número 30 , submetidas ou não ao tratamento térmico e a comparação com o valor crítico calculado de Tukey a $5 \%$.

\begin{tabular}{c|c|c|c|c}
\hline \hline Deflexão & & $\mathrm{KT}$ & $\mathrm{F}$ & $\mathrm{FT}$ \\
\hline \multirow{4}{*}{$30^{\circ}$} & $\mathrm{K}$ & $111,50 \mathrm{~s}$ & $107,67 \mathrm{~s}$ & $173,17 \mathrm{~s}$ \\
\cline { 2 - 5 } & $\mathrm{KT}$ & - & $3,83 \mathrm{~ns}$ & $61,67 \mathrm{~s}$ \\
\cline { 2 - 5 } & $\mathrm{F}$ & & - & $65,50 \mathrm{~s}$ \\
\hline \multirow{4}{*}{$45^{\circ}$} & & $\mathrm{KT}$ & $\mathrm{F}$ & $\mathrm{FT}$ \\
\cline { 2 - 5 } & $\mathrm{K}$ & $347,16 \mathrm{~s}$ & $162,00 \mathrm{~ns}$ & $292,50 \mathrm{~s}$ \\
\cline { 2 - 5 } & $\mathrm{KT}$ & - & $185,16 \mathrm{~ns}$ & $54,67 \mathrm{~ns}$ \\
\cline { 2 - 5 } & $\mathrm{F}$ & & - & $130,50 \mathrm{~ns}$ \\
\hline \multirow{4}{*}{$60^{\circ}$} & & $\mathrm{KT}$ & $\mathrm{F}$ & $\mathrm{FT}$ \\
\cline { 2 - 5 } & $\mathrm{K}$ & $457,92 \mathrm{~s}$ & $231,92 \mathrm{~ns}$ & $459,75 \mathrm{~s}$ \\
\cline { 2 - 5 } & $\mathrm{KT}$ & - & $244,00 \mathrm{~ns}$ & $16,17 \mathrm{~ns}$ \\
\cline { 2 - 5 } & $\mathrm{F}$ & & - & $227,83 \mathrm{~ns}$ \\
\hline \hline
\end{tabular}

Valor crítico de Tukey a $5 \%$ para $30^{\circ}=55,88$, para $45^{\circ}=190,03$ e para $60^{\circ}=260,38(\mathrm{~K}=$ tipo $\mathrm{K}$ não-tratada; $\mathrm{KT}=$ tipo $\mathrm{K}$ tratada;

$\mathrm{F}=$ Flexofile ${ }^{\circledR}$ não-tratada; FT $=$ Flexofile $^{\circledR}$ tratada; $\mathrm{ns}=$ não significante; $\mathrm{s}=$ significante). 
KAMEI, S. K.; SANTOS, M.; BOMBANA, A. C. Análise comparativa da flexibilidade de instrumentos endodônticos, submetidos ou não a tratamento térmico. Pesqui Odontol Bras, v. 14, n. 3, p. 213-218, jul./set. 2000.

não-tratados para todos os números e ângulos. A única exceção foi em relação à lima número 25 tratada, que, ao ser submetida à flexão de $45^{\circ}$, mostrou menor carga que a respectiva lima não-tratada.

A comparação das limas tipo $\mathrm{K}$ e Flexofile ${ }^{\circledR}$ não-tratadas proporcionou resultados diversos. Em algumas situações, os resultados foram semelhantes, em outras, houve diferenças estatisticamente significativas. Para as limas de número 15, a flexão nos ângulos determinados não denotou diferenças estatisticamente significantes, enquanto as limas Flexofile ${ }^{\circledR}$ não-tratadas de número 25 apresentaram menor resistência quando fletidas a $30^{\circ}$ e $45^{\circ}$ em comparação às limas tipo $\mathrm{K}$ não-tratadas correspondentes. Essas diferenças foram estatisticamente significativas ao nivel de $\alpha=5 \%$. Para a flexão a $60^{\circ}$, não houve diferença estatisticamente significante. Entre as limas tipo $\mathrm{K}$ e Flexofile ${ }^{\circledast}$ não-tratadas de número 30 , houveram diferenças significantes ao nivel de $\alpha=5 \%$ ao serem flexionadas à $30^{\circ}$. Quanto aos outros ângulos de flexão, não ocorreram diferenças.

A aplicação da carga na lima provoca uma deformação, a princípio chamada de elástica, pois a liga metálica por ser resiliente tem a capacidade de absorver essa energia e devolvê-la ao término de sua aplicação. Contudo, progredindo-se com a aplicação da carga, atinge-se o chamado limite de escoamento, início da fase plástica, e a lima deforma-se rapidamente. Isto é evidenciado por um grande alongamento, sem que haja o aumento da carga. Se o incremento da carga for mantido, as deformações continuarão até o momento da fratura. Essa capacidade do material de se deformar plasticamente e absorver energia antes de se fraturar é denominada de tenacidade ${ }^{7}$.

A diferença de resultados ao ensaio de flexão das limas tipo K tratadas e não-tratadas para todos os números e angulações foram significativas ao nível de $\alpha=5 \%$. As limas tipo K tratadas tiveram diminuição da rigidez, em decorrência da alteração do limite de escoamento que era atingido com uma carga menor, quando comparadas às limas do tipo $\mathrm{K}$ não-tratadas.

Os resultados, frente ao ensaio de flexão adotado, das limas Flexofile ${ }^{\circledR}$ tratadas, mostraram que o tratamento térmico não aumentou sua flexibilidade. Tal fato poderia ser esperado em função da presença de elementos metálicos que são adicionados à liga das limas Flexofile ${ }^{\circledR}$ que determinam a elevação da temperatura de recristalização ${ }^{3,5}$.
As diferenças estatisticamente significantes $(\alpha=5 \%)$ apresentadas pelas limas Flexofile ${ }^{\circledast}$ tratadas e não-tratadas de número 25 fletidas a $45^{\circ}$ são compreendidas ao analisarmos o limite de escoamento da lima tratada (que é atingido aplicando-se uma carga menor) quando comparada à lima não-tratada. Além disso, o limite apresenta-se com um intervalo maior, ou seja, mesmo aplicando-se uma diminuta carga, produzir-se-á uma deformação plástica bastante maior, comparativamente, à lima não-tratada. Tal fato não ocorreu na Flexofile ${ }^{\circledR}$ 25 ao fleti-la a $30^{\circ}$ em razão da probabilidade de haver uma relação entre a área da seção transversal e o tratamento térmico que influenciaria na diminuição da profundidade da recristalização, ou seja, caso o tratamento térmico não tenha sido suficiente para diminuir o nivel de energia interna acumulada, e se, além disso, o ângulo de $30^{\circ}$ não tiver sido suficiente para as limas Flexofile ${ }^{\circledR}$ tratadas e não-tratadas atingirem a fase de deformação plástica permanente. A flexão a $60^{\circ}$, tanto das limas Flexofile ${ }^{\circledR}$ tratadas como das não-tratadas, ao ultrapassarem o limite de escoamento, produziram deformações com cargas de valores semelhantes, pois o tratamento térmico não foi adequado para reduzir totalmente o nivel de encruamento por causa da presença de elementos metálicos, em diferentes concentrações ${ }^{3}$, que interferem no processo de recristalização elevando sua temperatu$\mathrm{ra}^{5}$.

As limas Flexofile ${ }^{\circledR}$ e tipo K não-tratadas de números 25 para os ângulos de $30^{\circ}$ e $45^{\circ}$ e número 30 para o ângulo de $30^{\circ}$ apresentaram valores de flexão com diferenças estatísticas ao nível de $\alpha=5 \%$. Tal fato pode ser explicado, pois apesar da maior dureza do aço proporcionado pelo esforço mecânico executado durante a obtenção de um número maior de espiras na lima Flexofile ${ }^{\circledR}$, comparativamente à tipo $\mathrm{K}$, a primeira é mais flexivel por apresentar área da secção transversal triangular menor que a quadrangular da lima tipo $\mathrm{K}^{4}$. No entanto, ao atingir-se o ângulo de $60^{\circ}$, o limite de elasticidade da lima Flexofile ${ }^{\circledR}$ número 25 justamente por ter seção transversal menor, teria sido transposto e a carga necessária para deformar as limas apresentou-se equivalente às tipo $\mathrm{K}$.

As limas de número 30 Flexofile $^{\circledR}$ e tipo $\mathrm{K}$ não-tratadas apresentaram comportamento semelhante, ao serem fletidas a $30^{\circ}$, em relação às de número 25 dos mesmos tipos quando fletidas a $30^{\circ}$ e $45^{\circ}$. Provavelmente, porque a área da seção transversal menor da lima Flexofile ${ }^{\circledR}$ em comparação a tipo K pode não ter sido suficiente para evi- 
KAMEI, S. K.; SANTOS, M.; BOMBANA, A. C. Análise comparativa da flexibilidade de instrumentos endodônticos, submetidos ou não a tratamento térmico. Pesqui Odontol Bras, v. 14, n. 3, p. 213-218, jul./set. 2000.

denciar diferenças nos valores das cargas. A superioridade da flexibilidade decorrente da menor área da seção transversal da lima Flexofile ${ }^{\circledR}$ provavelmente foi suprimida pelo fato de a lima de número 30 apresentar uma maior massa metálica e durante o processo de conformação plástica despendeu-se mais energia mecânica, aumentando o nivel de encruamento.

A semelhança de valores das cargas apresentadas pelas limas tipo $\mathrm{K}$ e Flexofile ${ }^{\circledast}$ de número 15 não-tratadas para fleti-las até os ângulos determinados deve-se à pequena área da seção transversal apresentada por ambos os tipos de limas ${ }^{6}$. Acrescenta-se também o fato de a lima tipo K ser obtida por um processo cujas alterações são menores em algumas das propriedades mecânicas que a Flexofile ${ }^{\circledR 4}$.

\section{CONCLUSÕES}

Em vista dos resultados obtidos, parece lícito concluir que:

1. o tratamento recristalizador diminuiu a resistência à flexão das limas tipo $\mathrm{K}$ de forma significativa para as numerações e angulações estudadas;

2. as limas Flexofile ${ }^{\circledR}$ sofreram redução da resistência à flexão, porém não estatisticamente significante em todas as condições experimentais, exceto para a lima número 25 tratada;

3. as limas tipo K são mais susceptiveis ao tratamento térmico recristalizador como se observou neste estudo, quando comparadas com limas Flexofile ${ }^{\circledR}$.

KAMEI, S. K.; SANTOS, M.; BOMBANA, A. C. Comparative analysis of the flexibility of endodontic instruments, submitted or not to thermal treatment. Pesqui Odontol Bras, v. 14, n. 3, p. 213-218, jul./set. 2000.

The aim of this study was to evaluate the bending resistance of 2 types of files: $\mathrm{K}$ and Flexofile ${ }^{\circledR}$, from different numbers submitted or not to a recrystallizing thermal treatment. A hundred and forty-four files were submitted to the bending test in a torquemeter with a scale. The results made evident that the recrystallizing thermal treatment was able to cause a decrease on the bending resistance of the K type files between 56.78 and $82.06 \%(\alpha=0.05 \%)$. The Flexofile ${ }^{\circledR}$ files presented a decrease on the bending resistance between 1.94 and $50.60 \%(\alpha=0.05 \%)$, in comparison with non-treated Flexofile ${ }^{\circledR}$ files, except for number 25. In conclusion, the recrystallizing thermal treatment, as proposed, reduces the bending resistance of $\mathrm{K}$ type files in a significant way, and of Flexofile ${ }^{\circledR}$ files in a less marked way.

UNITERMS: Dental instruments; Root canal preparation.

\section{REFERÊNCIAS BIBLIOGRÁFICAS}

1. ALLISON, D. A.; MICHELICH, R. J.; WALTON, R. E. The influence of master cone adaptation on the quality of the apical seal. J Endod, v. 7, n. 2, p. 61-69, Feb. 1981.

2. AUN, C. E. Avaliação, através de microscopia eletrônica de varredura, da limpeza da parede do canal radicular (terço apical), após o preparo químico-mecânico, tendo como fonte de variação o tipo e o número de uso dos instrumentos. São Paulo, 1985. 73 p. Tese (Doutorado), Faculdade de Odontologia, Universidade de São Paulo.

3. BOMBANA, A. C. Análise química (quantitativa) das ligas de aço inoxidável de alguns instrumentos endodônticos de diferentes tipos e procedências contribuição ao estudo. São Paulo, 1986. 73 p. Tese (Doutorado) - Faculdade de Odontologia, Universidade de São Paulo.

4. BOMBANA, A. C.; SANTOS, M. Estudo comparativo da dureza Rockwell de ligas de aço empregadas na confecção de instrumentos endodônticos de diferentes tipos e procedências. Rev XXV Jan, v. 35, n. 47-52, dez. 1987.

5. BRESCIANI FILHO, E.; ZAVAGLIA, C. A. C.; BUTTON, S. T. et al. Conformação plástica dos metais. 4. ed. Campinas: UNICAMP, 1991. 385 p.
6. CAMPS, J. J.; PERTOT, W. J. Relationship between file size and stiffness of stainless steel instruments. Endodont Dent Traumatol, v. 10, n. 6, p. 260-263, Dec. 1994.

7. CHIAVERINI, V. Tecnologia mecânica. 2. ed. São Paulo : McGraw-Hill, 1986. 266 p.

8. CRAIG, R. G.; PEYTON, F. A. Physical properties of carbon steel root canal files and reamers. Oral Surg, v. 15, n. 2, p. 213-226, Feb. 1962.

9. CRAIG, R. G.; PEYTON, F. A. Physical properties of stainless steel endodontic files and reamers. Oral Surg, v. 16, n. 2, p. 206-217, Feb. 1963.

10. CRAIG, R. G.; McILVAN, E. D.; PEYTON, F. A. Bending and torsional properties of endodontic instruments. Oral Surg, v. 25, n. 2, p. 239-254, Feb. 1968.

11. DOLAN, D. W.; CRAIG, R. G. Bending and torsion of endodontic files with rhombus cross sections. J Endod, v. 8, n. 6, p. 260-264, June 1982.

12. ELIAS, C. N.; DE BIASI, R. S.; CHEVITARESE, O. Influência do tratamento térmico no limite de escoamento de fios ortodônticos. Rev Bras Odontol, v. 50, n. 1, p. 29-32, jan./fev. 1993.

13. MANGONON Jr., P. L.; THOMAS, G. Structure and properties of thermal-mechanically treated 304 stainless steel. Met Trans, v. 1, p. 1587-1594, Jun. 1970.

14. MULLANEY, T. P. Instrumentation of finely curved canals. Dent Clin North Am, v. 23, n. 4, p. 575-593, Oct. 1979. 
KAMEI, S. K.; SANTOS, M.; BOMBANA, A. C. Análise comparativa da flexibilidade de instrumentos endodônticos, submetidos ou não a tratamento térmico. Pesqui Odontol Bras, v. 14, n. 3, p. 213-218, jul./set. 2000.
15. PAIVA, J. G.; ANTONIAZZI, J. H. Endodontia: bases para a prática clínica. 2. ed. São Paulo : Artes Médicas, 1988. $885 \mathrm{p}$.
16. PESCE, H. F. Análise comparativa in vitro da eficiência de corte de alguns instrumentos de uso endodôntico em função de seu tipo e procedência, número de uso e operador. (Contribuição ao estudo). São Paulo, 1984. 44 p. Tese (Doutorado) - Faculdade de Odontologia, Universidade de São Paulo.

17. SANTOS, M. Avaliação comparativa do comportamento, diante de ensaio de torção, de limas endodônticas de diferentes tipos, marcas e números, submetidas ou não a um tratamento térmico recristalizador. São
Paulo, 1994. 76 p. Tese (Doutorado) - Faculdade de Odontologia, Universidade de São Paulo.

18. SETO, B. G.; NICHOLLS, J. I.; HARRINGTON, G. W. Torsional properties of twisted and machined endodontic files. J Endod, v. 16, n. 8, p. 355-360, Aug. 1990.

19. WALIA, H.; BRANTLEY, W. A.; GERSTEIN, H. An initial investigation of the bending and torsional properties of nitinol root canal files. J Endod, v. 14, n. 7, p. 346-351, July 1988.

20. WEINE, F. S.; HEALEY, H. J.; GERSTEIN, H. et al. Pre-curved files and incremental instrumentation for root canal enlargement. J Can Dent Assoc, v. 70, n. 4, p. 155-157, Apr. 1970
Recebido para publicação em 03/01/00 Enviado para reformulação em 19/01/00 Aceito para publicação em 06/02/00

\section{EVENTOS INTERNACIONAIS}

\section{"European Association for Osseointegration"}

Data: 14 a 16 de setembro de 2000

Local: Amsterdam, The Netherlands

Informações: EAO 2000 Organizing Secretariat, BHS Congress Service, Mrs Carol Huntjens Vondelstraat 11, 1054 GC Amsterdam, The Netherlands. Fax: + 3120 412-0383, e-mail:eao2000@bhs.nl

\section{"International Symposium on Management Alternatives for Carious Lesions"}

Data: 15 a 17 de setembro de 2000

Local: Charleston, South Africa

Informações: Office of Continuing Professional Development, MUSC College of Health Professions, PO Box 250822, 19 Hagood Ave, Suite 910, Charleston, SC 29425.

Tel.: (800) 651-2926 ou (843) 792-3777, fax: (843) 792-1107, e-mail: chinnijw@musc.edu, site: http://www2.musc.edu/cl/home.htm

\section{"American Academy of Periodontology"}

Data: 17 a 20 de setembro de 2000

Local: Honolulu, Hawaii
Informações: AAP, 737 North Michigan Avenue, Suite 800, Chicago, Illinois 60611.

Fax: + 312 573-3225, site: www.perio.org

\section{"American Association of Oral and Maxillofacial Surgeons"}

Data: 20 a 23 de setembro de 2000

Local: San Francisco, California

Informações: AAOMS, 9700 West Bryn Mawr Avenue, Rosemont, Illinois 60018.

Fax: + 847 678-6286

\section{"American Dental Association"}

Data: 14 a 18 de outubro de 2000

Local: Chicago, Illinois

Informações: ADA, 211 East Chicago Avenue, Chicago, Illinois 60611. Fax: + 312 440-2707, site: www.ada.org

\section{"Greater New York Dental Meeting"}

Data: 26 a 29 de novembro de 2000

Local: New York, New York

Informações: GNY Dental Meeting, 1535 Broadway, 3rd Floor, New York, New York, 10036-4017. Fax: + 212 398-6922 